$11-1-2016$

\title{
E-Bayesian Estimation of the Parameter of the Logarithmic Series Distribution
}

Parviz Nasiri

University of Payam Noor, Tehran, Iran, pnasiri45@yahoo.com

Hassan Esfandyarifar

University of Payam Noor, Tehran, Iran

Follow this and additional works at: http://digitalcommons.wayne.edu/jmasm

Part of the Applied Statistics Commons, Social and Behavioral Sciences Commons, and the Statistical Theory Commons

\section{Recommended Citation}

Nasiri, Parviz and Esfandyarifar, Hassan (2016) "E-Bayesian Estimation of the Parameter of the Logarithmic Series Distribution," Journal of Modern Applied Statistical Methods: Vol. 15 : Iss. 2 , Article 37.

DOI: $10.22237 /$ jmasm/1478003700

Available at: http://digitalcommons.wayne.edu/jmasm/vol15/iss2/37

This Regular Article is brought to you for free and open access by the Open Access Journals at DigitalCommons@WayneState. It has been accepted for inclusion in Journal of Modern Applied Statistical Methods by an authorized editor of DigitalCommons@WayneState. 


\section{E-Bayesian Estimation of the Parameter of the Logarithmic Series Distribution}

Cover Page Footnote

$---$

This regular article is available in Journal of Modern Applied Statistical Methods: http://digitalcommons.wayne.edu/jmasm/vol15/ 


\section{E-Bayesian Estimation of the Parameter of the Logarithmic Series Distribution}

\section{Parviz Nasiri}

University of Payame Noor

Tehran, Iran
Hassan Esfandyarifar

University of Payame Noor

Tehran, Iran

E-Bayesian estimation is introduced to estimate the parameter of logarithmic series distribution. In addition, E-Bayesian, Bayesian and maximum likelihood estimation with through applying mean squared error.

Keywords: Logarithmic series distribution, E-Bayesian estimation, Bayesian estimation, maximum likelihood estimation, means squared error

\section{Introduction}

The logarithmic series distribution (LSD) is obtained by expanding the logarithmic function $-\log (1-\theta)$ as a power series in. Alternatively, it can also be derived as a limiting case of zero-truncated negative binomial distribution as $k$ decreases to zero. In either case, the logarithmic series distribution is a very useful distribution on the positive integers (Nasiri, 2011). Estimation is an important topic in statistical inference. Bayesian approach is an important approach in the estimation of parameter. A suitable prior distribution plays an effective role in reducing error in the estimation. Therefore, the more the prior information is obtained, the more it affects the posterior.

Lindley and Smith (1972) argued hierarchical prior. E-Bayesian estimation is another method introduced by Han and Ding (2004). Han (2005) applied EBayesian estimation for forecast of security investment. He also (2006, 2007) presented hierarchical Bayesian estimation for computing as well as E-Bayesian estimation for transition probability. In this study, maximum likelihood, Bayesian, and E-Bayesian estimations of the parameter of logarithmic series distribution are discussed in detail. This paper considers the maximum likelihood estimation of $\theta$,

Parviz Nasiri is in the Department of Statistics. Email them at: pnasiri45@yahoo.com. 


\section{ESTIMATION PARAMETER OF LSD}

the Bayesian estimation of $\theta$, and the E-Bayesian estimation of $\theta$; by use of a simulation, all estimations will be compared by MSE.

\section{Maximum Likelihood Estimation of $\theta$}

Let $\mathrm{f}(x)$ be the density of the logarithmic series distribution given by

$$
\mathrm{f}(x)=\frac{-1}{\log (1-\theta)} \cdot \frac{\theta^{x}}{x}, \quad x=1,2,3, \ldots, \quad 0<\theta<1
$$

The maximum likelihood estimation of $\theta$ in the above distribution is derived by i.i.d observations $x_{1}, x_{2}, \ldots x_{n}$. Hence, the likelihood function is given by

$$
1(\theta)=\left(\frac{1}{-\log (1-\theta)}\right)^{n} \cdot \frac{\theta^{n \bar{x}}}{\prod_{i=1}^{n} x_{i}}=(-\log (1-\theta))^{-n} \frac{\theta^{n \bar{x}}}{\prod_{i=1}^{n} x_{i}}
$$

Similarly, the logarithm of the likelihood function is given by

$$
\log 1(\theta)=n \bar{x} \log \theta-n \log (-\log (1-\theta))-\sum_{i=1}^{n} \log x_{i}
$$

There are two ways to estimate $\theta$. The first is to apply the "optimum" command in $\mathrm{R}$ software, and the second is to take the first order derivative of $\log 1(\theta)$ over $\theta$ and set it equal to zero, as in the following:

$$
\begin{aligned}
& \frac{d \log 1(\theta)}{d \theta}=0 \\
& \frac{\bar{x}}{\theta}+\frac{1}{(1-\theta)(\log (1-\theta))}=0 \\
& (1-\theta)^{1-\theta}=\mathrm{e}^{\frac{-\theta}{\bar{x}}}, \quad 0<\theta<1
\end{aligned}
$$

Assume $\mathrm{g}(\theta)=(1-\theta)^{1-\theta}-\mathrm{e}^{\frac{-\theta}{\bar{x}}}$. This equation can be solved via the NewtonRaphson method: 


\section{NASIRI \& ESFANDYARIFAR}

$$
\theta_{n}=\theta_{n-1}-\frac{\mathrm{g}\left(\theta_{n-1}\right)}{\dot{\mathrm{g}}\left(\theta_{n-1}\right)}
$$

where

$$
\dot{\mathrm{g}}\left(\theta_{n-1}\right)=(1-\theta)^{1-\theta}[-\log (1-\theta)-1]+\frac{\mathrm{e}^{\frac{-\theta}{\bar{x}}}}{\bar{x}},
$$

and Fixed-Point method as

$$
\theta_{n}=\mathrm{h}\left(\theta_{n-1}\right)
$$

such that

$$
\mathrm{h}(\theta)=\bar{x}(\theta-1) \log (1-\theta) .
$$

Equations (4) and (5) were solved using the MATLAB software. The "optimum" command was used in $\mathrm{R}$ software. There is additional discussion regarding the MLE logarithmic series in Bohning (1983).

\section{Bayesian Estimation of $\theta$}

Let $\pi(\theta)$ be prior density of $\theta$ that has beta distribution:

$$
\begin{gathered}
\pi(\theta)=\frac{1}{\mathrm{~B}(a, b)} \theta^{a-1}(1-\theta)^{b-1} \\
\mathrm{~B}(a, b)=\frac{\Gamma(a) \Gamma(b)}{\Gamma(a+b)}, \quad \Gamma(a)=\int_{0}^{\infty} t^{a-1} \mathrm{e}^{-t} d t
\end{gathered}
$$

By using i.i.d. observations $x_{1}, x_{2}, \ldots x_{n}$, the posterior distribution of $\theta$ was calculated as in the following:

$$
\pi(\theta \mid \underline{x})=\frac{1(\theta) \pi(\theta)}{\int_{0}^{1} 1(\theta) \pi(\theta) d \theta}=\frac{(-\log (1-\theta))^{-n} \theta^{n \bar{x}+a-1}(1-\theta)^{b-1}}{\int_{0}^{1}(-\log (1-\theta))^{-n} \theta^{n \bar{x}+a-1}(1-\theta)^{b-1} d \theta}
$$




\section{ESTIMATION PARAMETER OF LSD}

where $\mathrm{l}(\theta)$ is the likelihood function introduced in (2).

Note that $(-\log (1-\theta))^{-n}$ can be expanded as $\theta^{-n} \sum_{m=0}^{\infty} \rho_{m}(-n) \theta^{m}$, where $\rho 0(-n)=1, \rho_{m}(-n)=n \psi_{m-1}(m-n-1)$ for $m \geq 1$, and the coefficients $\psi m($.$) , are$ Sterling polynomials given by Castellares and Lemonte (2014).

Consider

$$
(-\log (1-\theta))^{-n} \theta^{n \bar{x}+a-1}(1-\theta)^{b-1}=\sum_{m=0}^{\infty} \rho_{m}(-n) \theta^{m+n(\bar{x}-1)+a-1}(1-\theta)^{b-1}
$$

and suppose

$$
\begin{aligned}
\mathrm{A}(a) & =\sum_{m=0}^{\infty} \rho_{m}(-n) \int_{0}^{1} \theta^{m+n(\bar{x}-1)+a-1}(1-\theta)^{b-1} d \theta \\
& =\sum_{m=0}^{\infty} \rho_{m}(-n) \mathrm{B}(m+n(\bar{x}-1)+a, b)
\end{aligned}
$$

Then

$$
\pi(\theta \mid \underline{x})=\frac{\sum_{m=0}^{\infty} \rho_{m}(-n) \theta^{m+n(\bar{x}-1)+a-1}(1-\theta)^{b-1}}{\mathrm{~A}(a)}
$$

The Bayesian estimation of $\theta$ under loss function $1(\theta, d)=(d-\theta)^{2}$ is $\mathrm{E}(\theta \mid \underline{x})$,

$$
\mathrm{E}(\theta \mid \underline{x})=\frac{\mathrm{A}(a+1)}{\mathrm{A}(a)}
$$

$\mathrm{E}(\theta \mid \underline{x})$ is computed by numerical methods using $\mathrm{R}$ software.

\section{E-Bayesian Estimation}

Let the prior distribution of $\theta$ be given as:

$$
\pi(\theta \mid a, b)=\frac{1}{\mathrm{~B}(a, b)} \theta^{a-1}(1-\theta)^{b-1}, \quad 0<\theta<1
$$




\section{NASIRI \& ESFANDYARIFAR}

where $a$ and $b$ are super parameters. According to Han (1997) $a$ and $b$ should be selected to guarantee $\pi(\theta \mid a, b)$ is a decreasing function of $\theta$. Therefore, we applied one order derivative of $\pi(\theta \mid a, b)$ over $\theta$ to obtain

$$
\frac{d \pi(\theta \mid a, b)}{d \theta}=\frac{\Gamma(\alpha+\beta)}{\Gamma(\alpha) \Gamma(\beta)} \theta^{a-2}(1-\theta)^{b-2}[(a-1)(1-\theta)-(b-1) \theta]
$$

Because $a>0, b>0$, and $0<\theta<1$, then $0<a \leq 1, b>1$ result in $\frac{d \pi(\theta \mid a, b)}{d \theta}<0$. Thus, $\pi(\theta \mid a, b)$ is a decreasing function of $\theta$ given $0<a \leq 1, b>1$.

As $b$ grows larger, the tail of the beta density function grows thinner. However, as far as the robustness of Bayesian estimation is concerned (Berger, 1985), the thinner-tailed prior distribution often leads to the worse robustness of the Bayesian estimate. Accordingly, $b$ should not be too big; it is better to be selected below the given upper bound $c(c>1)$ (see Han \& Ding, 2004). All in all, the super parameters $a$ and $b$ were selected to be in the ranges $0<a \leq 1$ and $1<b \leq c$.

Let $a=1$ and $b$ have density function given by the following:

$$
\mathrm{g}(b)=\frac{1}{c-1}, \quad 1<b \leq c
$$

Hence, the prior distribution is given by

$$
\begin{gathered}
\pi(\theta \mid a=1, b)=\pi(\theta \mid b)=b(1-\theta)^{b-1}, \quad 0<\theta<1 \\
\pi(\theta, b)=\pi(\theta \mid b) \mathrm{g}(b)=\frac{b}{c-1}(1-\theta)^{b-1}, \quad 0<\theta<1, \quad 1<b \leq c
\end{gathered}
$$

If the prior distribution is named $\pi_{E}(\theta)$, it is calculated as

$$
\pi_{E}(\theta)=\frac{1}{c-1} \int_{1}^{c} b(1-\theta)^{b-1} d b
$$

then $\pi(\theta \mid \underline{x})=\frac{1(\theta) \pi_{E}(\theta)}{\int_{0}^{1} 1(\theta) \pi_{E}(\theta) d \theta}$.

Consider 


\section{ESTIMATION PARAMETER OF LSD}

$$
\sum_{m=0}^{\infty} \rho_{m}(-n) \theta^{m+n(\bar{x}-1)+a-1} \int_{1}^{c} b(1-\theta)^{b-1} d b
$$

and suppose $\mathrm{B}(a)=\sum_{m=0}^{\infty}\left(\rho_{m}(-n) \int_{1}^{c} \mathrm{~B}(m+n(\bar{x}-1)+a, b) d b\right)$. Then

$$
\pi_{E}(\theta \mid \underline{x})=\frac{\sum_{m=0}^{\infty} \rho_{m}(-n) \theta^{m+n(\bar{x}-1)+a-1} \int_{1}^{c} b(1-\theta)^{b-1} d b}{\mathrm{~B}(a)}
$$

is the posterior distribution of $\theta$ and, under loss function $1(\theta, d)=(d-\theta)^{2}$, the Expected Bayesian (E-Bayesian) estimation is given as

$$
\mathrm{E}(\theta \mid \underline{x})=\frac{\mathrm{B}(a+1)}{\mathrm{B}(a)}
$$

$\mathrm{E}(\theta \mid \underline{x})$ is computed by numerical methods using $\mathrm{R}$ software.

\section{Simulation}

The simulation logarithmic series distribution is applied and the MSE among these three estimations are compared. The sample sizes chosen are $n=10$ (10)50, 100 from the logarithmic series distribution and then the above sampling is repeated

\begin{tabular}{|c|c|c|c|c|c|c|}
\hline \multirow[b]{2}{*}{$n$} & \multicolumn{2}{|c|}{ MLE } & \multicolumn{2}{|c|}{ BAYES } & \multicolumn{2}{|c|}{ E-BAYES } \\
\hline & $\hat{\boldsymbol{\theta}}$ & MSE & $\hat{\theta}$ & MSE & $\hat{\theta}$ & MSE \\
\hline 10 & 0.1754406 & 0.02280529 & 0.268888 & 0.015603929 & 0.2670986 & 0.015603929 \\
\hline 20 & 0.1848736 & 0.01274284 & 0.235789 & 0.009822050 & 0.2349044 & 0.009710344 \\
\hline 30 & 0.1888380 & 0.00885097 & 0.223687 & 0.007274929 & 0.2231012 & 0.007219458 \\
\hline 40 & 0.1915576 & 0.00665076 & 0.217965 & 0.005719935 & 0.2175264 & 0.005686960 \\
\hline 50 & 0.1951806 & 0.00536783 & 0.216286 & 0.004821819 & 0.2159331 & 0.004798442 \\
\hline 100 & 0.1968546 & 0.00251246 & 0.207595 & 0.002366069 & 0.2074195 & 0.002360269 \\
\hline
\end{tabular}
1000 times. In all the tables below, $a=1, b=1$.

Table 1. MSE of MLE, Bayesian, and E-Bayesian estimation for $c=1.1, \theta=0.2$ 


\section{NASIRI \& ESFANDYARIFAR}

Table 2. MSE of MLE, Bayesian, and E-Bayesian estimation for $c=1.5, \theta=0.2$

\begin{tabular}{|c|c|c|c|c|c|c|}
\hline \multirow[b]{2}{*}{$n$} & \multicolumn{2}{|c|}{ MLE } & \multicolumn{2}{|c|}{ BAYES } & \multicolumn{2}{|c|}{ E-BAYES } \\
\hline & $\hat{\theta}$ & MSE & $\hat{\theta}$ & MSE & $\hat{\boldsymbol{\theta}}$ & MSE \\
\hline 10 & 0.1754406 & 0.02280529 & 0.268888 & 0.015603929 & 0.2600561 & 0.013984288 \\
\hline 20 & 0.1848736 & 0.01274284 & 0.235789 & 0.009822050 & 0.2313332 & 0.009281856 \\
\hline 30 & 0.1888380 & 0.00885097 & 0.223687 & 0.007274929 & 0.2207135 & 0.007003590 \\
\hline 40 & 0.1915576 & 0.00665076 & 0.217965 & 0.005719935 & 0.2157289 & 0.005557730 \\
\hline 50 & 0.1951806 & 0.00536783 & 0.216286 & 0.004821819 & 0.2144824 & 0.004706188 \\
\hline 100 & 0.1968546 & 0.00251246 & 0.207595 & 0.002366069 & 0.2066942 & 0.002337212 \\
\hline
\end{tabular}

Table 3. MSE of MLE, Bayesian, and E-Bayesian estimation for $c=2, \theta=0.2$

\begin{tabular}{|c|c|c|c|c|c|c|}
\hline \multirow[b]{2}{*}{$n$} & \multicolumn{2}{|c|}{ MLE } & \multicolumn{2}{|c|}{ BAYES } & \multicolumn{2}{|c|}{ E-BAYES } \\
\hline & $\hat{\theta}$ & MSE & $\hat{\theta}$ & MSE & $\hat{\boldsymbol{\theta}}$ & MSE \\
\hline 10 & 0.1754406 & 0.02280529 & 0.268888 & 0.015603929 & 0.2517434 & 0.012635242 \\
\hline 20 & 0.1848736 & 0.01274284 & 0.235789 & 0.009822050 & 0.2269419 & 0.008802810 \\
\hline 30 & 0.1888380 & 0.00885097 & 0.223687 & 0.007274929 & 0.2177276 & 0.006756307 \\
\hline 40 & 0.1915576 & 0.00665076 & 0.217965 & 0.005719935 & 0.2134603 & 0.005407923 \\
\hline 50 & 0.1951806 & 0.00536783 & 0.216286 & 0.004821819 & 0.2126412 & 0.004597898 \\
\hline 100 & 0.1968546 & 0.00251246 & 0.207595 & 0.002366069 & 0.2057618 & 0.002309824 \\
\hline
\end{tabular}

Table 4. MSE of MLE, Bayesian, and E-Bayesian estimation for $c=3, \theta=0.2$

\begin{tabular}{|c|c|c|c|c|c|c|}
\hline \multirow[b]{2}{*}{$n$} & \multicolumn{2}{|c|}{ MLE } & \multicolumn{2}{|c|}{ BAYES } & \multicolumn{2}{|c|}{ E-BAYES } \\
\hline & $\hat{\theta}$ & MSE & $\hat{\theta}$ & MSE & $\hat{\boldsymbol{\theta}}$ & MSE \\
\hline 10 & 0.1754406 & 0.02280529 & 0.268888 & 0.015603929 & 0.2370938 & 0.010660721 \\
\hline 20 & 0.1848736 & 0.01274284 & 0.235789 & 0.009822050 & 0.2187640 & 0.008047442 \\
\hline 30 & 0.1888380 & 0.00885097 & 0.223687 & 0.007274929 & 0.2120299 & 0.006352388 \\
\hline 40 & 0.1915576 & 0.00665076 & 0.217965 & 0.005719935 & 0.2090714 & 0.005159055 \\
\hline 50 & 0.1951806 & 0.00536783 & 0.216286 & 0.004821819 & 0.2090494 & 0.004414182 \\
\hline 100 & 0.1968546 & 0.00251246 & 0.207595 & 0.002366069 & 0.2039068 & 0.002262655 \\
\hline
\end{tabular}

Table 5. MSE of MLE, Bayesian, and E-Bayesian estimation for $c=5, \theta=0.2$

\begin{tabular}{|c|c|c|c|c|c|c|}
\hline \multirow[b]{2}{*}{$n$} & \multicolumn{2}{|c|}{ MLE } & \multicolumn{2}{|c|}{ BAYES } & \multicolumn{2}{|c|}{ E-BAYES } \\
\hline & $\hat{\boldsymbol{\theta}}$ & MSE & $\hat{\boldsymbol{\theta}}$ & MSE & $\hat{\boldsymbol{\theta}}$ & MSE \\
\hline 10 & 0.1754406 & 0.02280529 & 0.268888 & 0.015603929 & 0.2145302 & 0.008617966 \\
\hline 20 & 0.1848736 & 0.01274284 & 0.235789 & 0.009822050 & 0.2051204 & 0.007182372 \\
\hline 30 & 0.1888380 & 0.00885097 & 0.223687 & 0.007274929 & 0.2021512 & 0.005863618 \\
\hline 40 & 0.1915576 & 0.00665076 & 0.217965 & 0.005719935 & 0.2012877 & 0.004851086 \\
\hline 50 & 0.1951806 & 0.00536783 & 0.216286 & 0.004821819 & 0.2025892 & 0.004176082 \\
\hline 100 & 0.1968546 & 0.00251246 & 0.207595 & 0.002366069 & 0.2004542 & 0.002201122 \\
\hline
\end{tabular}




\section{ESTIMATION PARAMETER OF LSD}

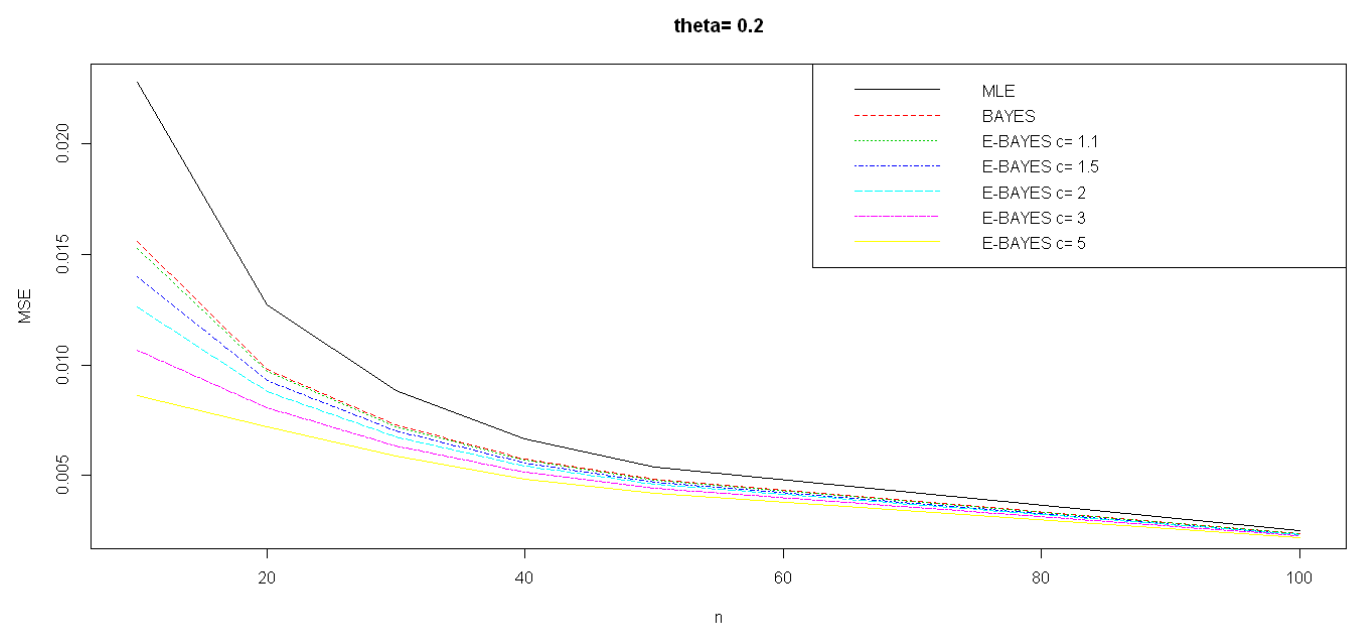

Figure 1. MSE of MLE, Bayesian, and E-Bayesian estimation for $\theta=0.2$

According to Tables 1-5 and Figure 1 below, if $\theta$ is close to zero, then the EBayesian estimator will be better than the others. Furthermore, the E-Bayesian estimator for big $\mathrm{c}$ is better than for that of small $\mathrm{c}$.

Table 6. MSE of MLE, Bayesian, and E-Bayesian estimation for $c=1.1, \theta=0.5$

\begin{tabular}{|c|c|c|c|c|c|c|}
\hline \multirow[b]{2}{*}{$n$} & \multicolumn{2}{|c|}{ MLE } & \multicolumn{2}{|c|}{ BAYES } & \multicolumn{2}{|c|}{ E-BAYES } \\
\hline & $\hat{\boldsymbol{\theta}}$ & MSE & $\hat{\theta}$ & MSE & $\hat{\boldsymbol{\theta}}$ & MSE \\
\hline 10 & 0.4493031 & 0.036068680 & 0.4727567 & 0.020631200 & 0.4703712 & 0.020689950 \\
\hline 20 & 0.4774472 & 0.015412300 & 0.4856296 & 0.011751570 & 0.4842737 & 0.011769950 \\
\hline 30 & 0.4822146 & 0.011401902 & 0.4872720 & 0.009491975 & 0.4863317 & 0.009503848 \\
\hline 40 & 0.4862952 & 0.007998191 & 0.4897325 & 0.006975903 & 0.4890090 & 0.006984277 \\
\hline 50 & 0.4899989 & 0.006229734 & 0.4925262 & 0.005593061 & 0.4919384 & 0.005597817 \\
\hline 100 & 0.4917995 & 0.003252503 & 0.4929799 & 0.003078263 & 0.4926775 & 0.003081360 \\
\hline
\end{tabular}

Table 7. MSE of MLE, Bayesian, and E-Bayesian estimation for $c=1.5, \theta=0.5$

\begin{tabular}{|c|c|c|c|c|c|c|}
\hline \multirow[b]{2}{*}{$n$} & \multicolumn{2}{|c|}{ MLE } & \multicolumn{2}{|c|}{ BAYES } & \multicolumn{2}{|c|}{ E-BAYES } \\
\hline & $\hat{\theta}$ & MSE & $\hat{\theta}$ & MSE & $\hat{\theta}$ & MSE \\
\hline 10 & 0.4493031 & 0.036068680 & 0.4727567 & 0.020631200 & 0.4611687 & 0.021062690 \\
\hline 20 & 0.4774472 & 0.015412300 & 0.4856296 & 0.011751570 & 0.4789428 & 0.011893800 \\
\hline 30 & 0.4822146 & 0.011401902 & 0.4872720 & 0.009491975 & 0.4826069 & 0.009577576 \\
\hline 40 & 0.4862952 & 0.007998191 & 0.4897325 & 0.006975903 & 0.4861318 & 0.007033491 \\
\hline 50 & 0.4899989 & 0.006229734 & 0.4925262 & 0.005593061 & 0.4895955 & 0.005627388 \\
\hline 100 & 0.4917995 & 0.003252503 & 0.4929799 & 0.003078263 & 0.4914670 & 0.003097037 \\
\hline
\end{tabular}




\section{NASIRI \& ESFANDYARIFAR}

Table 8. MSE of MLE, Bayesian, and E-Bayesian estimation for $c=2, \theta=0.5$

\begin{tabular}{|c|c|c|c|c|c|c|}
\hline \multirow[b]{2}{*}{$n$} & \multicolumn{2}{|c|}{ MLE } & \multicolumn{2}{|c|}{ BAYES } & \multicolumn{2}{|c|}{ E-BAYES } \\
\hline & $\hat{\boldsymbol{\theta}}$ & MSE & $\hat{\theta}$ & MSE & $\hat{\boldsymbol{\theta}}$ & MSE \\
\hline 10 & 0.4493031 & 0.036068680 & 0.4727567 & 0.020631200 & 0.4506494 & 0.021772010 \\
\hline 20 & 0.4774472 & 0.015412300 & 0.4856296 & 0.011751570 & 0.4726720 & 0.012145060 \\
\hline 30 & 0.4822146 & 0.011401902 & 0.4872720 & 0.009491975 & 0.4781747 & 0.009721149 \\
\hline 40 & 0.4862952 & 0.007998191 & 0.4897325 & 0.006975903 & 0.4826866 & 0.007126098 \\
\hline 50 & 0.4899989 & 0.006229734 & 0.4925262 & 0.005593061 & 0.4867802 & 0.005685544 \\
\hline 100 & 0.4917995 & 0.003252503 & 0.4929799 & 0.003078263 & 0.4900001 & 0.003122489 \\
\hline
\end{tabular}

Table 9. MSE of MLE, Bayesian, and E-Bayesian estimation for $c=3, \theta=0.5$

\begin{tabular}{|c|c|c|c|c|c|c|}
\hline \multirow[b]{2}{*}{$n$} & \multicolumn{2}{|c|}{ MLE } & \multicolumn{2}{|c|}{ BAYES } & \multicolumn{2}{|c|}{ E-BAYES } \\
\hline & $\hat{\boldsymbol{\theta}}$ & MSE & $\hat{\boldsymbol{\theta}}$ & MSE & $\hat{\boldsymbol{\theta}}$ & MSE \\
\hline 10 & 0.4493031 & 0.036068680 & 0.4727567 & 0.020631200 & 0.4329851 & 0.023664110 \\
\hline 20 & 0.4774472 & 0.015412300 & 0.4856296 & 0.011751570 & 0.4617896 & 0.012865210 \\
\hline 30 & 0.4822146 & 0.011401902 & 0.4872720 & 0.009491975 & 0.4703766 & 0.010129876 \\
\hline 40 & 0.4862952 & 0.007998191 & 0.4897325 & 0.006975903 & 0.4765777 & 0.007386216 \\
\hline 50 & 0.4899989 & 0.006229734 & 0.4925262 & 0.005593061 & 0.4817677 & 0.005854256 \\
\hline 100 & 0.4917995 & 0.003252503 & 0.4929799 & 0.003078263 & 0.4873595 & 0.003187286 \\
\hline
\end{tabular}

Table 10. MSE of MLE, Bayesian, and E-Bayesian estimation for $c=5, \theta=0.5$

\begin{tabular}{|c|c|c|c|c|c|c|}
\hline \multirow[b]{2}{*}{$n$} & \multicolumn{2}{|c|}{ MLE } & \multicolumn{2}{|c|}{ BAYES } & \multicolumn{2}{|c|}{ E-BAYES } \\
\hline & $\hat{\boldsymbol{\theta}}$ & MSE & $\hat{\theta}$ & MSE & $\hat{\theta}$ & MSE \\
\hline 10 & 0.4493031 & 0.036068680 & 0.4727567 & 0.020631200 & 0.4081765 & 0.027960910 \\
\hline 20 & 0.4774472 & 0.015412300 & 0.4856296 & 0.011751570 & 0.4460818 & 0.014636860 \\
\hline 30 & 0.4822146 & 0.011401902 & 0.4872720 & 0.009491975 & 0.4590000 & 0.011149126 \\
\hline 40 & 0.4862952 & 0.007998191 & 0.4897325 & 0.006975903 & 0.4676159 & 0.008033505 \\
\hline 50 & 0.4899989 & 0.006229734 & 0.4925262 & 0.005593061 & 0.4744028 & 0.006284713 \\
\hline 100 & 0.4917995 & 0.003252503 & 0.4929799 & 0.003078263 & 0.4834468 & 0.003338421 \\
\hline
\end{tabular}




\section{ESTIMATION PARAMETER OF LSD}

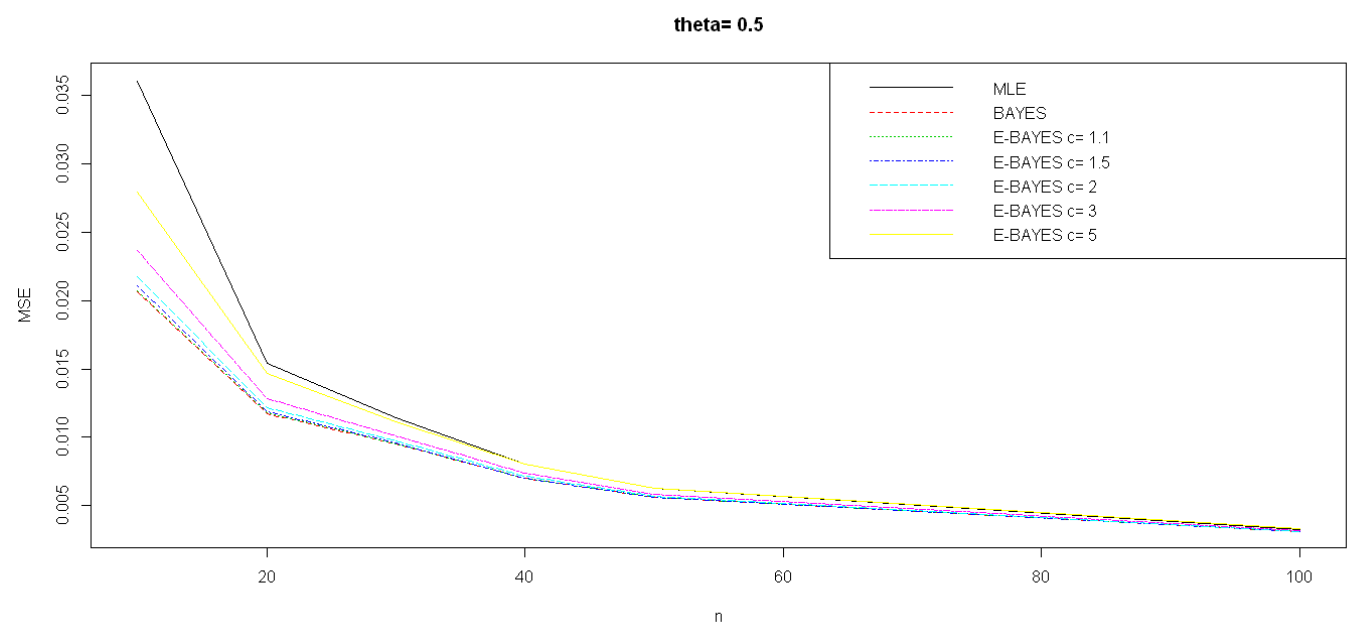

Figure 2. MSE of MLE, Bayesian, and E-Bayesian estimation for $\theta=0.5$

According to Tables 6-10 and Figure 2 above, if $\theta$ is equal to 0.5 , then the Bayes estimator will be better than the others.

Table 11. MSE of MLE, Bayesian, and E-Bayesian estimation for $c=1.1, \theta=0.8$

\begin{tabular}{|c|c|c|c|c|c|c|}
\hline \multirow[b]{2}{*}{$n$} & \multicolumn{2}{|c|}{ MLE } & \multicolumn{2}{|c|}{ BAYES } & \multicolumn{2}{|c|}{ E-BAYES } \\
\hline & $\hat{\theta}$ & MSE & $\hat{\theta}$ & MSE & $\hat{\theta}$ & MSE \\
\hline 10 & 0.7565883 & 0.016160921 & 0.7342053 & 0.016276950 & 0.7320425 & 0.016636190 \\
\hline 20 & 0.7771566 & 0.006733548 & 0.7635732 & 0.007190623 & 0.7623963 & 0.007302307 \\
\hline 30 & 0.7853821 & 0.004234158 & 0.7757980 & 0.004486893 & 0.7749946 & 0.004538776 \\
\hline 40 & 0.7908123 & 0.003149407 & 0.7834313 & 0.003279836 & 0.7828241 & 0.003307885 \\
\hline 50 & 0.7891018 & 0.002431975 & 0.7831037 & 0.002570948 & 0.7826102 & 0.002592876 \\
\hline 100 & 0.7962759 & 0.000991319 & 0.7931716 & 0.001020993 & 0.7929747 & 0.001032422 \\
\hline
\end{tabular}

Table 12. MSE of MLE, Bayesian, and E-Bayesian estimation for $c=1.5, \theta=0.8$

\begin{tabular}{|c|c|c|c|c|c|c|}
\hline \multirow[b]{2}{*}{$n$} & \multicolumn{2}{|c|}{ MLE } & \multicolumn{2}{|c|}{ BAYES } & \multicolumn{2}{|c|}{ E-BAYES } \\
\hline & $\hat{\theta}$ & MSE & $\hat{\theta}$ & MSE & $\hat{\theta}$ & MSE \\
\hline 10 & 0.7565883 & 0.016160920 & 0.7342053 & 0.016276950 & 0.7240587 & 0.018094940 \\
\hline 20 & 0.7771566 & 0.006733548 & 0.7635732 & 0.007190623 & 0.7580292 & 0.007758596 \\
\hline 30 & 0.7853821 & 0.004234158 & 0.7757980 & 0.004486893 & 0.7720108 & 0.004751512 \\
\hline 40 & 0.7908123 & 0.003149407 & 0.7834313 & 0.003279836 & 0.7805691 & 0.003423867 \\
\hline 50 & 0.7891018 & 0.002431975 & 0.7831037 & 0.002570948 & 0.7807726 & 0.002682152 \\
\hline 100 & 0.7962759 & 0.000991319 & 0.7931716 & 0.001020993 & 0.7919732 & 0.001041411 \\
\hline
\end{tabular}




\section{NASIRI \& ESFANDYARIFAR}

Table 13. MSE of MLE, Bayesian, and E-Bayesian estimation for $c=2, \theta=0.8$

\begin{tabular}{|c|c|c|c|c|c|c|}
\hline \multirow[b]{2}{*}{$n$} & \multicolumn{2}{|c|}{ MLE } & \multicolumn{2}{|c|}{ BAYES } & \multicolumn{2}{|c|}{ E-BAYES } \\
\hline & $\hat{\theta}$ & MSE & $\hat{\theta}$ & MSE & $\hat{\theta}$ & MSE \\
\hline 10 & 0.7565883 & 0.016160920 & 0.7342053 & 0.016276950 & 0.7156517 & 0.019879590 \\
\hline 20 & 0.7771566 & 0.006733548 & 0.7635732 & 0.007190623 & 0.7534337 & 0.008318997 \\
\hline 30 & 0.7853821 & 0.004234158 & 0.7757980 & 0.004486893 & 0.7688793 & 0.005013464 \\
\hline 40 & 0.7908123 & 0.003149407 & 0.7834313 & 0.003279836 & 0.7782094 & 0.003568182 \\
\hline 50 & 0.7891018 & 0.002431975 & 0.7831037 & 0.002570948 & 0.7788405 & 0.002790142 \\
\hline 100 & 0.7962759 & 0.000991319 & 0.7931716 & 0.001020993 & 0.7910271 & 0.001068188 \\
\hline
\end{tabular}

Table 14. MSE of MLE, Bayesian, and E-Bayesian estimation for $c=3, \theta=0.8$

\begin{tabular}{|c|c|c|c|c|c|c|}
\hline \multirow[b]{2}{*}{$n$} & \multicolumn{2}{|c|}{ MLE } & \multicolumn{2}{|c|}{ BAYES } & \multicolumn{2}{|c|}{ E-BAYES } \\
\hline & $\hat{\boldsymbol{\theta}}$ & MSE & $\hat{\boldsymbol{\theta}}$ & MSE & $\hat{\theta}$ & MSE \\
\hline 10 & 0.7565883 & 0.016160920 & 0.7342053 & 0.016276950 & 0.7034408 & 0.023040870 \\
\hline 20 & 0.7771566 & 0.006733548 & 0.7635732 & 0.007190623 & 0.7469579 & 0.009294010 \\
\hline 30 & 0.7853821 & 0.004234158 & 0.7757980 & 0.004486893 & 0.7645488 & 0.005464724 \\
\hline 40 & 0.7908123 & 0.003149407 & 0.7834313 & 0.003279836 & 0.7749910 & 0.003818176 \\
\hline 50 & 0.7891018 & 0.002431975 & 0.7831037 & 0.002570948 & 0.7762116 & 0.002971987 \\
\hline 100 & 0.7962759 & 0.000991319 & 0.7931716 & 0.001020993 & 0.7897346 & 0.001107678 \\
\hline
\end{tabular}

Table 15. MSE of MLE, Bayesian, and E-Bayesian estimation for $c=5, \theta=0.8$

\begin{tabular}{|c|c|c|c|c|c|c|}
\hline \multirow[b]{2}{*}{$n$} & \multicolumn{2}{|c|}{ MLE } & \multicolumn{2}{|c|}{ BAYES } & \multicolumn{2}{|c|}{ E-BAYES } \\
\hline & $\hat{\boldsymbol{\theta}}$ & MSE & $\hat{\boldsymbol{\theta}}$ & MSE & $\hat{\theta}$ & MSE \\
\hline 10 & 0.7565883 & 0.016160921 & 0.7342053 & 0.016276950 & 0.6907121 & 0.027424820 \\
\hline 20 & 0.7771566 & 0.006733548 & 0.7635732 & 0.007190623 & 0.7410871 & 0.010504326 \\
\hline 30 & 0.7853821 & 0.004234158 & 0.7757980 & 0.004486893 & 0.7609354 & 0.005989118 \\
\hline 40 & 0.7908123 & 0.003149407 & 0.7834313 & 0.003279836 & 0.7724500 & 0.004101652 \\
\hline 50 & 0.7891018 & 0.002431975 & 0.7831037 & 0.002570948 & 0.7741743 & 0.003164136 \\
\hline 100 & 0.7962759 & 0.000991319 & 0.7931716 & 0.001020993 & 0.7888172 & 0.001145513 \\
\hline
\end{tabular}




\section{ESTIMATION PARAMETER OF LSD}

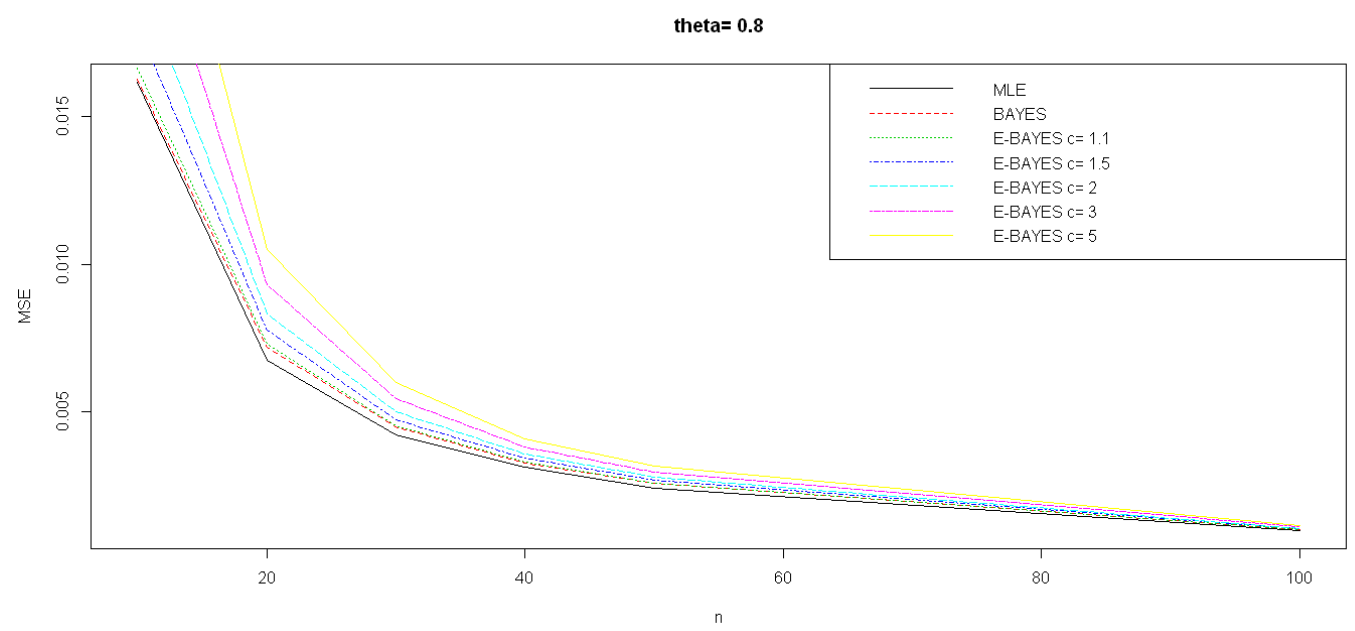

Figure 3. MSE of MLE, Bayesian, and E-Bayesian estimation for $\theta=0.8$

According to Tables 11-15 and Figure 3 above, if $\theta$ is close to 1 , then the maximum likelihood estimator will be better than the others.

\section{Conclusion}

The comparison among the three estimators revealed that with increasing sample size, all three estimators come together and as a result, the error rate is reduced. However, in the small samples according to the value of $\theta$ is superior to any of the rest, of the figures and tables is shown.

\section{References}

Berger, J. O. (1985). Statistical decision theory and Bayesian analysis (2nd ed.). New York, NY: Springer-Verlag.

Bohning, D. (1983). Maximum likelihood estimation of the logarithmic series distribution. Statistics Hefte, 24(1), 121-140. doi: 10.1007/BF02932495

Castellares, F., \& Lemonte, A. J. (2014). A new generalized Weibull distribution generated by gamma random variables. Journal of the Egyptian Mathematical Society, 23(2), 382-390. doi: 10.1016/j.joems.2014.03.009 


\section{NASIRI \& ESFANDYARIFAR}

Han, M. (1997). The structure of hierarchical prior distribution and its application. Operations Research and Management Science, 6(3), 31-40. Available from http://en.cnki.com.cn/Journal_en/A-A002-YCGL-1997-03.htm

Han, M. (2005). Expected Bayesian method for forecast of security investment. Operations Research and Management Science, 14(5), 98-102. Available from http://en.cnki.com.cn/Article_en/CJFDTotalYCGL200505020.htm

Han, M. (2006). E-Bayesian estimation and hierarchical Bayesian estimation of estate probability. Operations Research and Management Science, 15(5), 7074. Available from http://en.cnki.com.cn/Article_en/CJFDTOTALYCGL200605013.htm

Han, M. (2007). E-Bayesian estimation of failure probability and its application. Mathematical and Computer Modeling, 45(9-10), 1272-1279. doi: 10.1016/j.mcm.2006.11.007

Han, M. (2009). E-Bayesian estimation and hierarchical Bayesian estimation of rate. Applied Mathematical Modeling, 33(4), 1915-1922. doi: 10.1016/j.apm.2008.03.019

Han, M., \& Ding, Y. (2004). Synthesized expected Bayesian method of parametric estimate. Journal of Systems Science and Systems Engineering, 13(1), 98-111. doi: 10.1007/s11518-006-0156-0

Lindley, D. V., \& Smith, A. F. M. (1972). Bayesian estimates for the linear model. Journal of the Royal Statistical Society. Series B (Methodological), 34(1), 1-41. Available from http://www.jstor.org/stable/2985048

Nasiri, P. (2011). Estimation parameter of zero truncated mixed Poisson models. International Journal of Mathematical Analysis, 5(10), 465-470.

Retrieved from http://www.m-hikari.com/ijma/ijma-2011/ijma-9-12-

2011/nasiriIJMA9-12-2011.pdf 